\title{
Advance preparation in task-switching: converging evidence from behavioral, brain activation, and model-based approaches
}

\author{
Frini Karayanidis ${ }^{1,2 *}$, Sharna Jamadar ${ }^{1,2}$, Hannes Ruge ${ }^{3}$, Natalie Phillips ${ }^{4}$, Andrew Heathcote $^{5}$ and \\ Birte U. Forstmann ${ }^{6}$
}

Functional Neuroimaging Laboratory, School of Psychology and Centre for Brain and Mental Health Research, University of Newcastle, Newcastle, NSW, Australia

2 Schizophrenia Research Institute, Sydney, NSW, Australia

3 Neuroimaging Center, Department of Psychology, Institute of General Psychology, Biopsychology and Methods of Psychology, Technische Universitat Dresden, Dresden, Germany

4 Department of Psychology, Concordia University, Montreal, OC, Canada

5 Newcastle Cognition Laboratory, School of Psychology, Centre for Brain and Mental Health Research, University of Newcastle, Newcastle, NSW, Australia

${ }^{6}$ Spinoza Center for Neuroimaging, Department of Psychology, Universiteit van Amsterdam, Amsterdam, Netherlands

\section{Edited by:}

Shulan Hsieh, National Cheng Kung

University, Taiwan

\section{Reviewed by:}

Nachshon Meiran, Ben-Gurion

University, Israel

Myeong-Ho Sohn, George Washington

University, USA

\section{*Correspondence:}

Frini Karayanidis, Functional

Neuroimaging Laboratory, School of

Psychology, University of Newcastle,

University Drive, NSW 2308, Australia.

e-mail: frini.karayanidis@newcastle.

edu.au
Recent research has taken advantage of the temporal and spatial resolution of event-related brain potentials (ERPs) and functional magnetic resonance imaging (fMRI) to identify the time course and neural circuitry of preparatory processes required to switch between different tasks. Here we overview some key findings contributing to understanding strategic processes in advance preparation. Findings from these methodologies are compatible with advance preparation conceptualized as a set of processes activated for both switch and repeat trials, but with substantial variability as a function of individual differences and task requirements. We then highlight new approaches that attempt to capitalize on this variability to link behavior and brain activation patterns. One approach examines correlations among behavioral, ERP and fMRI measures. A second "model-based" approach accounts for differences in preparatory processes by estimating quantitative model parameters that reflect latent psychological processes. We argue that integration of behavioral and neuroscientific methodologies is key to understanding the complex nature of advance preparation in task-switching.

Keywords: advance preparation, task-switching, ERP, fMRI, evidence accumulation models

\section{INTRODUCTION}

Goal-directed behavior is an aspect of executive control that requires both the ability to maintain focus on a single goal and to switch attention among alternative goals. The taskswitching paradigm experimentally manipulates the strategic and automatic processes that affect advance preparation for, and implementation of, a switch or repeat in task. Strategic processes include proactive and reactive task adjustments (e.g., Rogers and Monsell, 1995), whereas automatic processes include associative task cue and target priming (e.g., Wylie and Allport, 2000). The cued-trials paradigm can temporally dissociate the relative contributions of preparatory and target-driven processes on task-switching performance by providing control over the onset and duration of the preparation interval (Figure 1). In the present paper, we are particularly interested in the processes that occur during this preparation interval, and hence, we focus largely on studies using this paradigm ${ }^{1}$. Although we will refer to relevant behavioral findings, a comprehensive review

Abbreviations: ACC, anterior cingulate cortex; BOLD, blood-oxygen-level-dependent; CTI, cue-target interval; DLPFC, dorsolateral prefrontal cortex; ERP, event-related brain potentials; fMRI, functional magnetic resonance imaging; FPC, frontopolar cortex; PFC, prefrontal cortex; PM, premotor cortex; PPC, posterior parietal cortex; PreSMA, pre-supplementary motor areas; RT, response times; SMA, supplementary motor areas; STN, subthalamic nucleus; VLPFC, ventrolateral prefrontal cortex. of behavioral studies is both outside the scope of this paper and the focus of an independent parallel paper by Kiesel et al. (in press). As conventional behavioral measures, like mean response times (RT) and error rates, represent the endpoint of decision making, they do not offer a direct insight about the temporal evolution of the processes leading up to the decision. This limitation is particularly pertinent with respect to processes contributing to advance preparation, as these processes are more removed from the final response. Yet, it is precisely the ability to tap into advance preparation processes that has made the task-switching paradigm particularly attractive as a measure of goal-directed behavior. The concept of preparation incorporates advance selection, activation, and maintenance of task-relevant perceptual and motor representations and control of interference from task-irrelevant representations (Jennings and van der Molen, 2005).

${ }^{1}$ In choosing to focus on studies using the cued-trials paradigm, we are not implying that other paradigms are less informative for studying task-switching. However, we argue that with many other paradigms (e.g., alternating runs, pair-wise task-switching, variants of the Wisconsin Card Sorting Test) it is harder to differentiate processes associated with advance preparation from other processes (e.g., evaluation of the response to the previous trial, interpretation of feedback information and updating of context based on response and feedback) and to precisely time-lock ERP averaging to the onset of any preparation processes. 


\section{Cued-trials task switching paradigm}

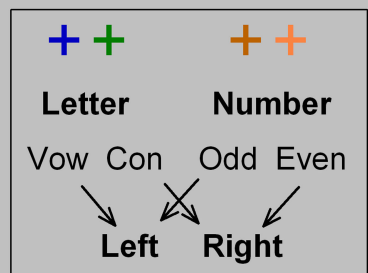

\section{Informative Cue}

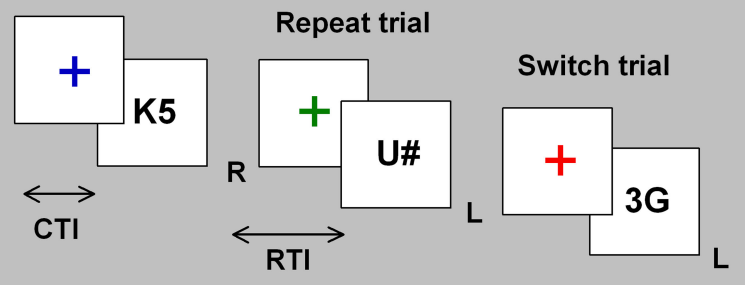

Non-informative Cue

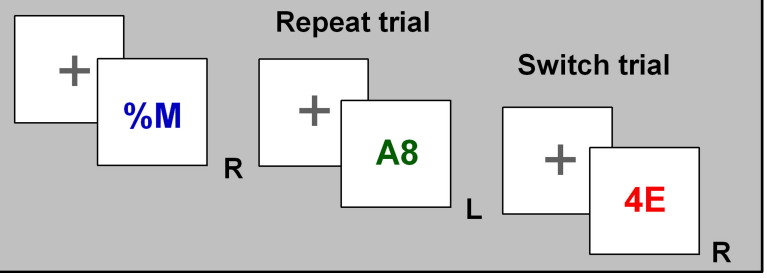

FIGURE 1 | Cued trials task-switching paradigms include a random sequence of switch and repeat trials with each stimulus preceeded by a cue. Cues signal either the task to be performed on the subsequent target stimulus (task cues) or whether the task will change or repeat upon the subsequent stimulus (transition cues). The task-switching paradigm involves switching between two or more simple tasks defined on the basis of stimulus-set, response-set, or both. Typically, performance is poorer (e.g., slower RT, more errors) on trials that require a change (switch trials) than a repeat task (repeat trials) - a phenomenon commonly referred to as switch cost. Cued trials paradigms include cue-target sequences where a cue carries task-specific (Task A or B) or trial transition (switch or repeat) information prior to target onset and therefore allow presentation of random sequences of switch and repeat. Increasing either cue-target interval (CTI) or responsetarget interval (RTI) results in a reduction in switch cost, providing evidence for active preparation and passive dissipation processes, respectively (Meiran, Chorev \& Sapir, 2000). Many cued trials paradigms use task cueing and map one cue to each task (1:1 cue-to-task mapping) so that a task switch is confounded with a cue change (Logan \& Bundesen, 2003). Recent studies use two cues per task (2:1 cue-to-task mapping, as shown above) to separate task switching from cue change effects. We have focused here only on studies using the cued-trials paradigm as it provides tight control over preparation onset on a trial by trial basis.

Given the inherent limits of performance data, many taskswitching studies have applied neuroscientific research methodologies to provide a window into the processes contributing to the overt behavioral outcome (see also Kiesel et al., in press). The excellent temporal resolution of event-related brain potentials (ERP; Rugg and Coles, 1995) can differentiate between activity that is timelocked to cue onset vs. target onset, thereby offering a direct measure of preparatory and target-driven processes, respectively. The excellent spatial resolution of functional magnetic resonance imaging (fMRI; Logothetis, 2002) can identify neural circuits involved in preparing and implementing a switch and repeat trial. In this focused review, we first present a selective overview of some key findings that highlight the contributions that ERP and $\mathrm{fMRI}^{2}$ methodologies have made to our understanding of the nature of strategic processes in advance preparation, and how specific areas within the fronto-parietal network may underpin these processes. ERP and fMRI methodologies have different strengths with regard to answering questions about when preparatory processes occur and where they take place in the human brain, respectively. Therefore, for each methodology we focus on three different questions that it is particularly suited to address. We argue that despite the wealth of information provided by ERP and fMRI research, meaningful insights into how advance preparation in task-switching is accomplished requires reconciliation of behavioral and multiple neuroscientific approaches (see also Hommel and Colzato, 2010). In the second part of this focused review, we discuss some recent approaches to this reconciliation. One approach involves mapping individual variability in performance onto variability in temporally distinct ERP components and the strength of BOLD fMRI signals. A second approach involves mapping neuroscientific measures to the latent parameters of quantitative evidence accumulation decision-process models of behavior. These recent approaches offer insights into the role of strategic processes in advance preparation in task-switching that are not available from any individual methodology alone.

\section{ERP INDICES OF ADVANCE PREPARATION PROCESSES}

Cue-locked ERP waveforms for task repeat and task-switch trials show a similar overall morphology, but are differentially modulated by task parameters including: the amount of information provided by the cue, the overlap between stimulus- or response-sets for the different tasks, and trial context effects. Typically, switch trials show a larger cue-locked posterior positivity than repeat trials, which we refer to as the differential switch positivity and modulation of a fronto-central pre-target negativity. The larger centro-parietal positivity for switch as compared to repeat trials has been very consistently reported but inconsistently labeled in the task-switching literature. We use the term "cue-locked positivity" to refer to the P3b-like component seen in cue-locked waveforms for both switch and repeat trials and the term "differential switch positivity" to refer to the relative increase in this positivity for cue-locked switch trials. The morphology of the fronto-centrally maximal negativity differs across studies, possibly reflecting differences arising from the use of a different recording reference. For the purposes of this review, we primarily focus on the slow frontal negativity seen mainly with a common average reference, unless specified otherwise. Here we focus on three questions that illustrate how cue-locked ERPs have contributed to defining the nature of strategic processes in advance preparation.

Do cued-trials paradigms measure cue-repetition benefit or task-switch cost? Or, in other words, do behavioral differences between switch and repeat trials arise simply because the 1:1

${ }^{2}$ Note that several recent studies have applied other neuroscience methodologies, including transcranial magnetic stimulation (Rushworth et al., 2002) and patient lesions (Rushworth et al., 2003). We have chosen to focus on ERP and fMRI methodologies that have been more widely used in this field. 
mapping between cue and task produces a confound between cue change and task change (e.g., Logan and Bundesen, 2003)? ERP studies comparing 1:1 and 2:1 cue-to-task mappings show that both cue change and task change affect preparatory processes in task-switching (Figure 1 caption). Switching between color and form cues that were mapped to the same task resulted in cue change effects on an early negativity, the N2 (e.g., Nicholson et al., 2006a), that were not seen when shifting between two letter cues (Jost et al., 2008). The cue-locked posterior positivity was affected by both cue change and task change (Perianez and Barcelo, 2009), but cue change had a smaller (Jost et al., 2008) and briefer (Nicholson et al., 2006a) effect. So, more automatic processes associated with a change in cue feature affected early cue-locked ERP that are linked to stimulus feature processing. More strategic processes associated with cue-task remapping and task-set activation affected later components. Hence, ERP effects during the preparation interval provide strong evidence that with 1:1 mapping, both cue-repetition benefit and preparation to switch task may affect performance outcome, supporting the use of 2:1 cue-task mappings in cued-trials paradigms when the focus is on strategic preparatory processes.

Does advance preparation act on stimulus-sets, response-sets or both? ERP studies show distinct timelines and scalp distributions of cue-locked components depending on whether advance preparation involves remapping of stimulus-set and/or response-set. In task-switching paradigms, targets can be univalent (i.e., include exemplars from only one stimulus-set, such as A\# for letter task) or bivalent (i.e., include stimuli mapped to both tasks, such as A4 for letter task). Response-sets can also be bivalent (i.e., include overlapping mappings between stimuli and responses as shown above in Figure 1) or univalent (i.e., include either a single response choice for both tasks (e.g., same vs. different) or distinct stimulusresponse (S-R) mapping for each task (e.g., index and middle fingers of left hand = letter task, index and middle fingers of right hand = number task).

Using a brief cue-target interval (CTI) and task cues that persisted after target onset, Poulsen et al. (2005) found no difference in switch positivity between univalent and bivalent stimulus-sets that were mapped to bivalent responses, indicating that the switch positivity is not affected by stimulus valence when there is little opportunity or need for advance preparation (see also Karayanidis et al., 2003). Kieffaber and Hetrick (2005) compared switching between tasks and modalities using two visual matching tasks mapped to the same bivalent target stimuli (e.g., object shape vs. size matching tasks) and a univalent auditory frequency discrimination task. The same univalent response-set was used for all three tasks (e.g., match vs. mismatch response). Spatiotemporal principal components analysis produced three components within the latency range of the switch positivity. Two components were differentially sensitive to the sensory features of the stimulus-sets (i.e., switching between the three stimulus types and switching between the two visual stimulus features, respectively), whereas the third reflected generic switching regardless of stimulus modality or visual feature. As the tasks involved identical responses, these effects could not be associated with response-set preparation. Thus, with brief cues and long preparation intervals, the switch positivity appears to be sensitive to stimulus-set manipulations. Miniussi et al. (2005) found that the scalp distribution of the switch positivity did not differ when switching between tasks that are processed in neurally distinct areas (verbal vs. spatial tasks) supporting the existence of a common task-independent source for the switch positivity. These studies indicate that the switch positivity consists of many underlying components, at least one of which reflects a common task-non-specific preparation process. Paradigms that manipulate cue informativeness also support the existence of multiple switch positivities associated with stimulus-set preparation (Nicholson et al., 2006b; Karayanidis et al., 2009; see Figure 5 for details of the paradigm). Cues indicating that the task will change, but not necessarily specifying which task it will change to, elicited an early switch positivity. Cues that also identified the task to be switched to, elicited an additional later switch positivity. Together, these findings indicate that the switch positivity is comprised of a number of underlying components that reflect different processes associated with stimulus-set preparation. However, the switch positivity does not merely represent a simple shift in attentional spotlight to the relevant target feature (e.g., number vs. letter), as it is also elicited in preparation for switching between distinct tasks defined on the same stimulus-set (e.g., parity and magnitude of a single digit, Nicholson et al., 2006b). Astle et al. (2008) varied S-R mappings for identical bivalent stimulus-sets (e.g., A, a, G, g) with either univalent or bivalent response-sets. A large switch positivity emerged for both univalent and bivalent S-R mappings, but was prolonged in the latter condition, indicating that the duration of the switch positivity was affected by the need to remap stimulusset to response-set. Interestingly, unlike the frontal negativity discussed below, this prolonged switch positivity did not vary as a function of whether the task defined an overt or a covert (e.g., mental counting) response. These findings strongly suggest that the switch positivity consists of a number of subcomponents that are sensitive to shifting attention between different stimulus features, stimulus-sets and S-R mapping and is affected by the degree to which the upcoming target is prone to interference.

The late, slow frontal negativity in the cue-locked ERP appears to be sensitive to response-set preparation processes. Astle et al. (2008) found that the negativity was larger for switch than repeat trials when tasks required an overt response, but was absent when no overt response was required (e.g., in a mental counting task). This negativity has been shown to differ as a function of response valence. Mueller and colleagues found that the negativity was elicited exclusively by bivalent but not univalent response-sets (Mueller et al., 2007, 2009), whereas Astle et al. (2008) reported that it was larger for bivalent than univalent response-sets. So this negativity appears to be affected by the specific type of response categoryeffector mappings (e.g., a bivalent vs. univalent mapping; Mueller et al., 2007; Astle et al., 2008). The amplitude of this negativity is also affected by whether an overt response is required on the current trial and whether or not an overt response was made on the previous trial (Astle et al., 2006), consistent with the argument that response-sets are inhibited for no-go trials (Jamadar et al., 2010a). The evidence suggests that this late frontal negativity may be associated with preparatory remapping of effectors to response category. Together, these studies provide strong evidence that both stimulussets and response-sets, as well as the mapping between them, are prepared within the CTI and that these processes are mapped onto topographically and temporally distinct ERP components. 
Does task repetition involve advance preparation and, if so, does it entail similar processes to task-switching? Some models of taskswitching assume that the set of a completed task remains in a state of readiness (e.g., Rogers and Monsell, 1995) or dissipates only slowly over time (e.g., Wylie and Allport, 2000) so that, with typical CTIs, repeat trials provide a relatively inert baseline against which to assess switch preparation. Yet both behavioral and ERP studies that compare repeat trials in mixed-task blocks (i.e., typical cued-trials switching that involve mixture of switch and repeat trials) against all-repeat trials (i.e., trials in a single task or pure block) question this assumption. Although repeat and all-repeat trials differ only in context, repeat trials have a longer RT than all-repeat trials (i.e., a mixing cost). During the preparation interval, repeat trials show a small cue-locked positivity in the same time range as the differential switch positivity, whereas all-repeat trials show no cue-locked positivity (e.g., Goffaux et al., 2006; Ruge et al., 2006; Jost et al., 2008; Wylie et al., 2009). Non-informative cues, which indicate the timing of the upcoming target but do not carry information about task identity, also show no cue-locked positivity (e.g., Hsieh and Yu, 2003; Swainson et al., 2006; Jamadar et al., 2010a). The differential posterior positivity for repeat cues, compared to allrepeat and non-informative cues, is consistent with some type of preparation occurring even for repeat trials in a mixed-task block. This may occur because, in mixed-task blocks, the repeat-task-set would have been recently suppressed and reactivated. Hence, repeat trials may require more attentional control than all-repeat trials in order to reduce interference from the irrelevant task-set, albeit less so than switch trials. In support, Wylie et al. (2009) found differences in both activation and topography among these three trial types. The topography of the cue-locked positivity differed between all-repeat and repeat trials, but not significantly between repeat and switch trials. In contrast, the strength of activation progressively increased from all-repeat to repeat to switch trials. Notably, their Figure 5 also shows that parietal sources were activated for both switch and repeat trials, whereas frontal sources were activated only for switch trials. These findings suggest that repeat and switch trials in a mixed-task block share some preparation processes, whereas other processes may be selectively activated for switch trials only.

In summary, different cue-locked ERP components are associated with cue processing, stimulus-set preparation and response-set preparation. The cue-locked positivity and slow frontal negativity provide direct measures of anticipatory control in relation to stimulus-set and response-set selection, respectively. These components are compatible with the conceptualization of advance preparation as a set of processes that may be activated for switch and/or repeat trials depending on task parameters and requirements and that may include both central as well as modality and/or task-specific components (Jennings and van der Molen, 2005). ERP components measure differences in relative activation of these processes and their contribution to performance outcomes.

\section{LINKING FRONTO-PARIETAL NETWORKS TO PREPARATORY AND TARGET-DRIVEN CONTROL PROCESSES}

Consistent with the pivotal role of a fronto-parietal network in goaldirected behavior (e.g., Corbetta and Shulman, 2002; Braver and Ruge, 2006), many fMRI studies applying the task-switching paradigm report activation in a distributed network encompassing dorsolateral and ventrolateral aspects of the prefrontal cortex (DLPFC,
VLPFC), supplementary and pre-supplementary motor areas (SMA, pre-SMA), anterior cingulate cortex (ACC), and superior and inferior aspects of the posterior parietal cortex (PPC). However, there is large variability in the precise localization (see Figure 2A) and in the presence and strength of switch-related activations within this network (e.g., Wager et al., 2004), likely reflecting variability related to task parameters and strategy selection (see Figure 2B). In this section, we discuss fMRI studies that have addressed three questions regarding the role of the fronto-parietal network in task-switching.

Is there evidence for different networks supporting advance preparation and target-driven processes? Models of functional organization of the PFC argue that anterior and dorsal regions are involved in goal-directed control, whereas ventral regions are involved in target-driven control adjustments (Corbetta and Shulman, 2002; Koechlin and Summerfield, 2007). Studies that examine differences in the localization and strength of fMRI activation as a function of parametric modulation of the timing and opportunity for advance preparation have attempted to disentangle the contributions of preparatory and target-driven control processes (see Figure 2B caption). There is converging evidence that distinct areas of frontal and parietal cortex are differentially associated with preparatory and target-driven control during task-switching. Wylie et al. (2006) compared preparation-related activity in two tasks: one task was associated with highly effective preparation (i.e., as indicated by no residual switch cost after a long CTI) and the other with less effective preparation (i.e., as indicated by a significant residual cost). The highly prepared task showed switch-repeat activity differences during the preparation interval in DLPFC and in task-relevant regions, indicating that preparation served to "preactivate" task-relevant regions. In contrast, the less prepared task showed no switch-repeat activation difference in either frontal or task-relevant areas. ERP-fMRI correlations (Figure 3) showed that differential DLPFC activity for prepared vs. unprepared trials (averaged over switch and repeat) was associated with cue-locked ERP activity early in the preparation interval and before the emergence of switch-repeat activity differences in the superior PPC (sPPC) (Jamadar et al., 2010b). However, consistent with our earlier conclusion based on ERP data that preparation is not necessarily exclusive to switching, Sakai and Passingham (2006) found that larger frontopolar cortex (FPC) activity during task-specific preparation was associated with larger activity in task-selective regions after target onset and faster RT. The above findings are compatible with a FPC/ DLPFC role in goal-directed activation during advance preparation, leading to activation of task-relevant neural areas and performance facilitation.

There is also emerging evidence that VLPFC and premotor $(\mathrm{PM}) /$ pre-SMA activation may be associated with target-driven control. Badre and Wagner (2006) showed that switch-repeat activity differences in VLPFC decreased with increasing preparation interval (i.e., VLPFC activity was larger on unprepared than prepared trials). Jamadar et al. (2010b) found greater VLPFC activity in non-informative than informative cue blocks. Given the VLPFC's role in inhibition and/or interference control (e.g., Aron et al., 2004; Forstmann et al., 2008a), these findings are consistent with VLPFC activation providing target-driven interference control under unprepared conditions. PM/pre-SMA activity also shows variability associated with target-driven control 


\section{A Fronto-Parietal Activity in Switch $>$ Repeat Contrasts in Task-Switching}
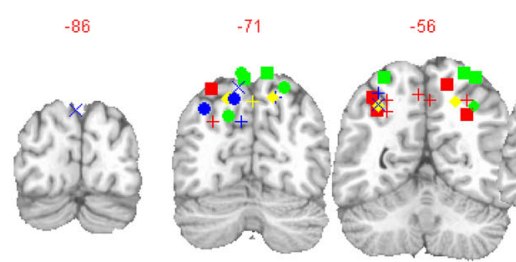

$-41$

$-26$

$-11$

4

19

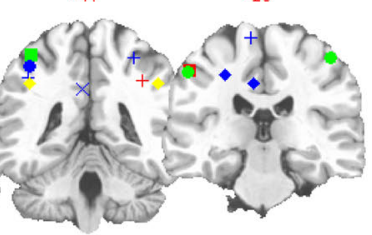

34

49
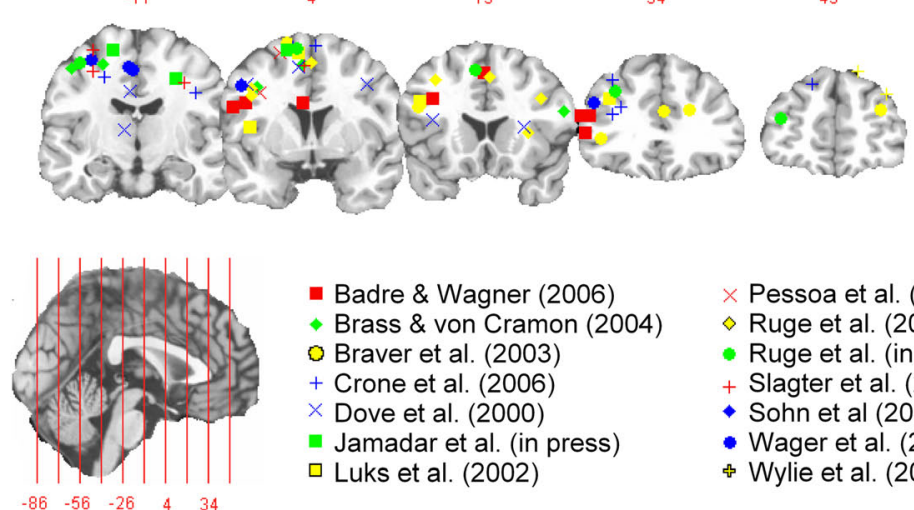

- Badre \& Wagner (2006)

$\times$ Pessoa et al. (2009)

- Brass \& von Cramon (2004)

$\checkmark$ Ruge et al. (2005)

Braver et al. (2003)

+ Crone et al. (2006)

+ Slagter et al. (2006)

$x$ Dove et al. (2000)

- Sohn et al (2000)

- Jamadar et al. (in press)

- Wager et al. (2004)

$\begin{array}{lllll}-86 & -56 & -26 & 4 & 34\end{array}$

$\square$ Luks et al. (2002)

\#Wylie et al. (2006)

\section{B Time-course of cue- and target-related activity relative to BOLD response}

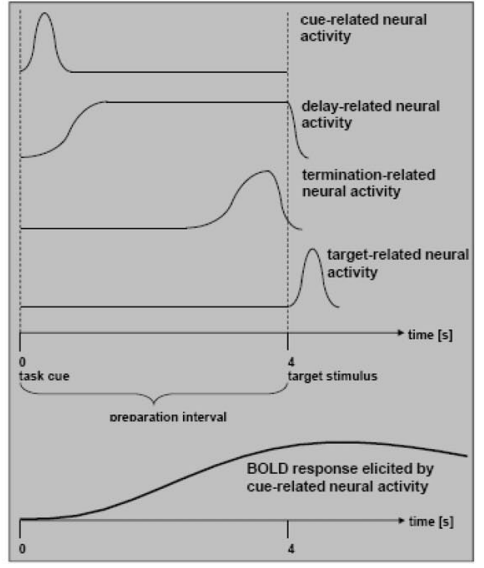

FIGURE 2 | Functional Magnetic Resonance Imaging (fMRI) studies measure a blood oxygenation-level dependent (BOLD) signal that is related to complex changes in blood flow, blood volume, and oxygenated and deoxygenated haemoglobin (see Logothetis, 2002 for a review). fMRI has superior spatial resolution; thus, it provides important information on the neuroanatomy of cognitive processing. (A) Fronto-parietal activity in switch $>$ repeat contrasts in cued task-switching studies, independent of CTI manipulation. Studies were included a) if they included at least one CTI condition that is considered long enough for advance preparation (i.e., CTI>500ms) but not long enough to require maintenance of a prepared state, and b) if they reported Talairach or MNI coordinates for significant Swt-Rpt activity. Hotspots indicate the peak of activity in each cluster reported in each study. Peaks that fall between the presented slices are shown on the closest corresponding slice. It may be seen that while there is variation in the precise localization of switch > repeat activity, there is also a considerable degree of consistency, with DLPFC, VLPFC, PM, pre-SMA and SPPC showing consistent activation. (B) fMRI studies addressing the concept of advance preparation face the challenge of how to disentangle two or more temporally overlapping BOLD responses, i.e., one or more responses that are associated with preparatory activity and one or more responses associated with target-related activity. Although no technique offers a complete solution to this challenge, a number of different approaches have been used in the task-switching literature: a) Early studies used a constant long CTI (e.g. 8s, Kimberg, Aguirre \& D'Esposito, 2000) to unambiguously link BOLD activity to cue- and target-related periods. For task switching paradigms, such long intervals might be problematic as the preparatory processes of interest might be masked by other intervening processes that are not under experimental control, such as maintenance and decay of preparatory state (e.g. Rogers \& Monsell, 1995; Jennings \& van der Molen, 2005b) Many studies use a partial trials design, where cue presence and target presence are orthogonally manipulated to precisely disentangle preparation- and target-related activity in cue-only trials (see Ruge, Goschke, \& Braver, 2009b). However, target omission can potentially elicit no-go responses (Jamadar et al., 2010a) introducing an unintended manipulation; c) A third approach varies the CTI and extracts BOLD responses associated with cue-related, delay-related, and target-related neural activity by introducing a separate model regressor that is coupled to the length of the CTI (e.g. Bunge, Kahn, Wallis, Miller \& Wagner, 2003). To obtain robust estimates, the CTI is typically varied across a relatively wide range (e.g., 4-12 s), which as mentioned above is problematic in task-switching paradigms; d) Purely experimental approaches such as multivariate pattern classification (e.g. Bode \& Haynes, 2009) has been used to identify brain regions that appear to express spatial activation patterns specific to certain information that is available during selective time periods during the trial. These new approaches need to be further explored. 


\section{Relationship Between RT-fMRI and ERP-fMRI Outcomes}

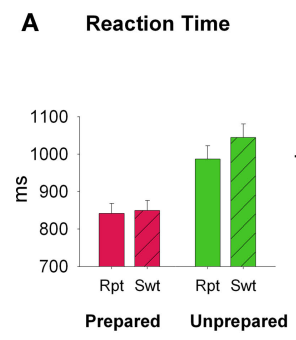

B Cue-Locked ERPs

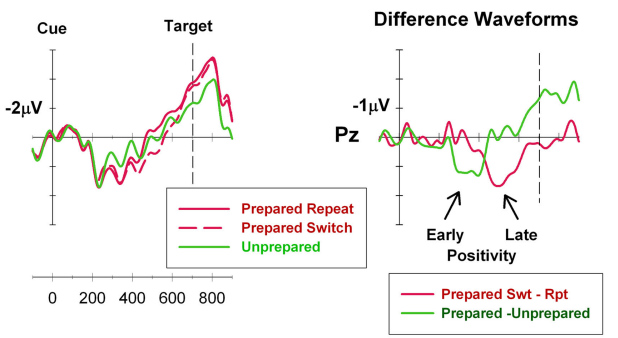

C Cue-Locked ERP seeded dipole modeling

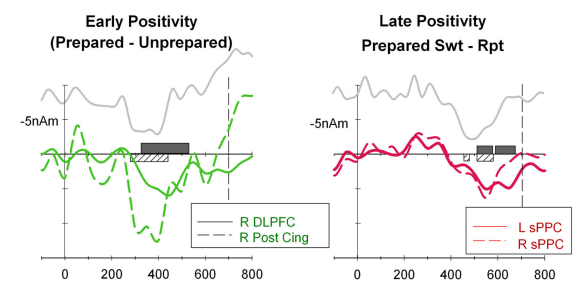

\section{Prepared > Unprepared fMRI Contrast}
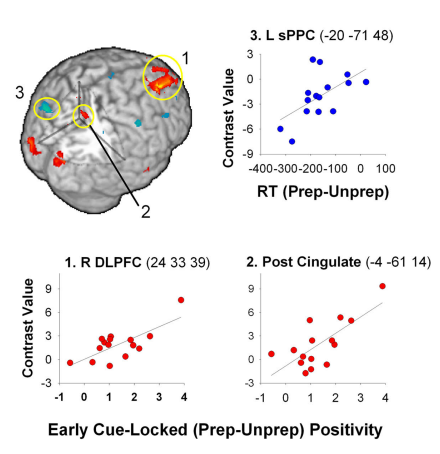

FIGURE 3 | Event-related brain potentials (ERP) are extracted from the electroencephalogram (EEG) through a process of signal averaging and

filtering. ERPs represent time-varying scalp fields that result from summation of post-synaptic electromagnetic activity generated by neuronal populations in different parts of the brain (Otten \& Rugg, 2005). While the location of neuronal generators of ERP activity cannot be directly inferred from scalp recorded activity (Rugg \& Coles, 1995), ERPs have excellent temporal resolution providing millisecond accuracy in differentiation between different conditions. Jamadar et al. (2010b) recorded ERP and fMRI using identical paradigms and the same participants on separate occasions. In this cued-trials paradigm (see Figure 1), prepared and unprepared trials were randomly presented with CTI-700ms. Prepared trials included an informative task cue followed by a bivalent target. Unprepared trials included a non-informative cue followed by an informative bivalent target. (A third condition was included on which an informative cue was followed by a target that was not mapped to any response and is discussed in Jamadar et al. (2010b)). (A) RT and RT switch cost were larger on prepared than unprepared trials. (B) Cue-locked ERP waveforms showed an early parietal positivity for prepared vs. unprepared trials, followed by a later parietal positivity for prepared switch vs. prepared repeat trials. Difference waveforms highlight the early positivity for both informative cues and the later positivity for switch cues only. (C) The relationships between ERP and fMRI outcomes (see D) were used to constrain seeded dipole models for ERP source analysis. This approach can identify potential generators of the ERP effect of interest and is the most common approach to multimodal integration (Dale \& Halgren, 2001). In this study, seeded dipole analysis revealed that the DLPFC and posterior cingulate were plausible generators of the early positivity for informative cues and the

\section{E Prepared Switch > Repeat fMRI Contrast}
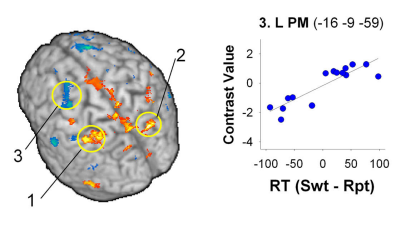

$$
\begin{aligned}
& \text { 1. L sPPC (-28-51 62) 2. R sPPC (20 -75 52) }
\end{aligned}
$$

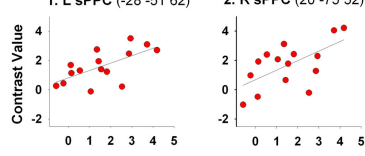

$$
\begin{aligned}
& \text { Late Cue-Locked (Swt - Rpt) Positivity }
\end{aligned}
$$

SPPC was a plausible generator for the later positivity for informative switch cues. (D and E) RT-fMRI and ERP-fMRI correlations. RT-fMRI correlations were used to examine neural activity directly related to the preparation and execution of a response. ERP-fMRI correlations allowed the temporal information inherent in the ERP measure to generate hypotheses about the timing of the processes reflected in $\mathrm{fMRI}$ activity. For example, these correlations show that DLPFC activation (see D1) is associated with activity occurring earlier in the CTI than SPPC activation (see E1 and E2). This type of distinction would be difficult to achieve using a traditional $\mathrm{fMRI}$ approach. Note that ERP-fMRI correlations provide quantitatively different information to a traditional $\mathrm{fMRI}$ contrast and can result in different regions of significant activation to more traditional fMRI contrasts. This is because individual variability in the BOLD signal is included in the error variance component of traditional fMRI contrasts whereas correlational analyses reveal important dimensions in BOLD signal variability that are related to inter-subject variation in RT and ERPs. Thus, correlational analyses can be used to infer, albeit indirectly, a relationship between specific cortical regions and sub-processes of the task, but it will only detect processes that show substantial individual differences between subjects (see also Forstmann et al., $2008 a, b)$. On the other hand, regions of activation that are evident in the contrast analyses but are absent from the correlational analyses may reflect either effects that have minimal individual variation or sub-processes of the task that are unrelated to the specific RT and ERP measures that are chosen to correlate. Thus correlations between RT, ERP and $\mathrm{AMRI}$ represent an opportunity to examine individual variability in $\mathrm{fMRI}$ contrasts and a method for using the temporal information in the ERP signal to examine the temporal dynamics of the fMRI data. 


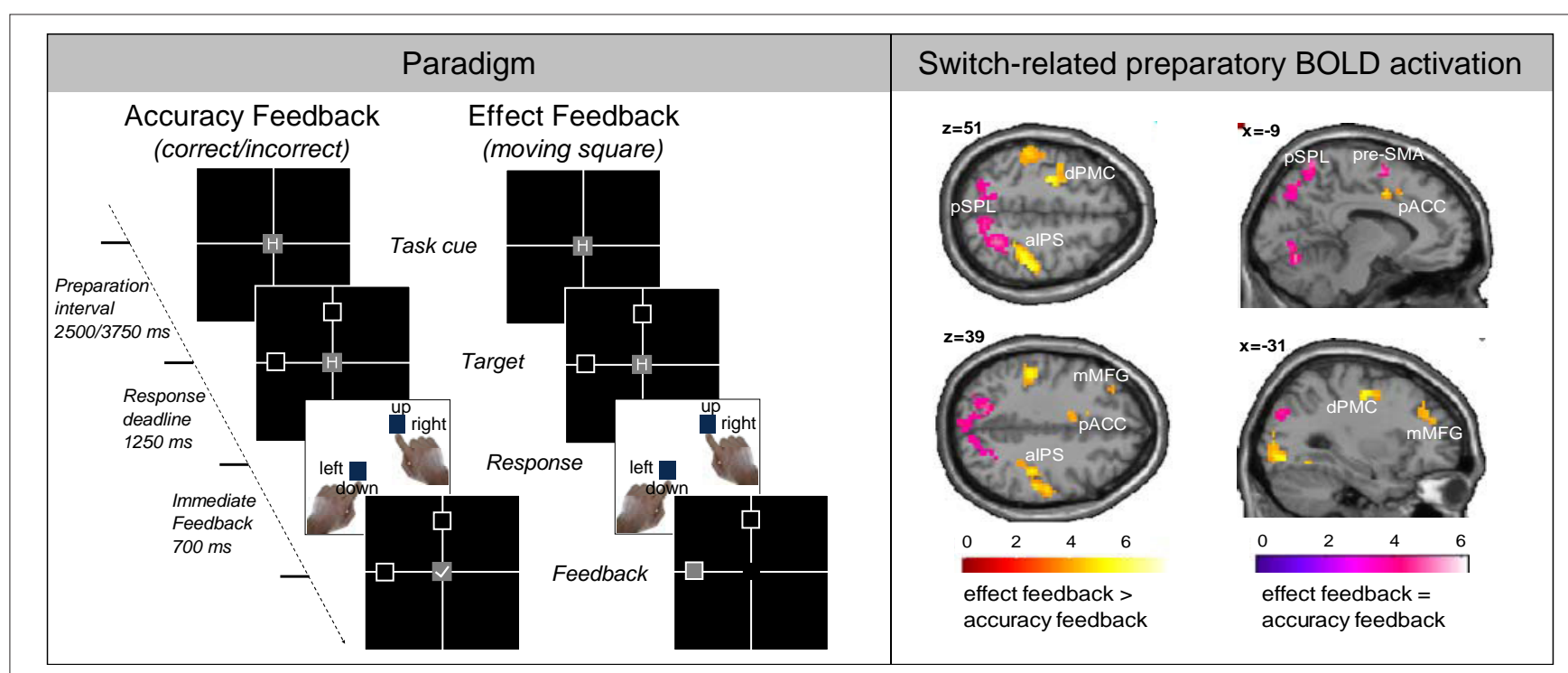

FIGURE 4 | From Ruge et al. (in press). (A) In this cued-trials paradigm, participants ( $n=18$ ) performed two blocked task-switching conditions involving either "accuracy feedback" or "effect feedback" after responding. On each trial, the currently relevant task was indicated by a centrally displayed task cue ("H" for horizontal discrimination and "V" for vertical discrimination). (B) Two types of preparatory BOLD activations were identified, associated either with "intentional preparatory control" processes (stronger switch-related activation in the effect-feedback condition than in the accuracy feedback condition) colored in red/yellow or with "attentional preparatory control" processes (similar switch-related activation for both feedback types) colored in blue/pink. processes. Switch-related PM/pre-SMA activity was found in unprepared (short CTI) but not prepared (long CTI) trials (e.g., Brass and von Cramon, 2004; Ruge et al., 2005). Although this effect lacks regional specialization (i.e., it is also evident at other fronto-parietal regions e.g., Ruge et al., 2005), it is consistent with the finding that individual variability in residual RT switch cost was positively correlated with switch-related activation selectively in PM (Jamadar et al., 2010b). So, participants who did not effectively prepare showed larger switch-related activation in PM. Interestingly, Slagter et al. (2006) found that reducing trial type probability for either switch or repeat trials also resulted in greater PM/pre-SMA activity for the corresponding trial type, suggesting that less practiced and/or less expected trial types show greater activation in PM regions. Together these results suggest that unprepared or inefficiently prepared trials, which require greater target-driven control to reduce interference in stimulus processing and response selection, are associated with greater activation in VLPFC and PM/pre-SMA than prepared trials. This effect is not necessarily specific to switch trials; rather it may be related to task parameters that increase the difficulty of the trial, regardless of its type, as these more difficult trials can benefit from advance preparation, or in its absence, require greater target-driven control. Alternatively, the finding that cues indicating the infrequent task yield higher activation in these areas could reflect an increase in the amount of evidence required for a response (the "response threshold") for these less practiced and expected trial types. However, using a different experimental approach to differentiate preparatory and target-driven activations, Ruge et al. (2009a) question such a strong separation of domain-independent control (as measured by cue-related activation) and concrete task implementation (as measured by targetrelated activation). Specifically, the same posterior prefrontal and parietal areas that were engaged by task cues (irrespective of switch or repeat) were reactivated by the subsequent target and these same areas exhibited preparatory activation when the target stimulus was presented in advance of the cue.

Is there evidence for switch-specific activation in the frontoparietal network? Many of the above studies also show switchrelated activation in PPC, but this region is affected by different task parameters than PFC. For example, Badre and Wagner (2006) reported dissociation between switch-related activation in frontal and parietal regions. Increased preparation was associated with reduced switch-repeat activation in mid-VLPFC but increased activation in inferior PPC (iPPC). Moreover, mid-VLPFC and iPPC activation were mapped onto different computational model parameters related to control of stimulus and response conflict, respectively. These findings suggest a compensatory balance between preparatory and target-driven control adjustments, such that greater switch-related preparatory control at the long CTIs (indexed by greater iPPC activity) may result in less interference control being required when the target appears (indexed by reduced VLPFC activity).

The variation in parietal switch-related activation with CTI found by Badre and Wagner (2006) is consistent with switch-repeat activation differences in parietal cortex during preparation. Jamadar et al. (2010b) found switch-repeat activation differences in the sPPC for prepared trials but not for unprepared trials. Braver et al. (2003) found that the strength of sPPC activation varied with the size of the RT switch cost, so that greater activity in this region was associated with a smaller residual RT switch cost (i.e., more effective preparation to switch). This is consistent with stronger switch-repeat activation in the sPPC during preparation resulting in stronger preparatory adjustment processes. If sPPC activation instead reflected target-driven adjustments, it should have been 
associated with larger residual switch cost (i.e., less effective preparation). Consistent with this interpretation, Wylie et al. (2006) found switch-repeat activation in sPPC for a fully-prepared task but not for a less prepared task. However, other studies report the exact opposite pattern, that is, stronger switch-repeat activation in sPPC for unprepared trials as compared to prepared trials (i.e., Brass and von Cramon, 2004; Ruge et al., 2005). The significant positive correlation of switch-repeat activation with the amplitude of the cue-locked switch positivity, but not with stimulus-locked ERP components, provides more direct support for a specific role of the sPPC in preparatory but not in target-driven control processes (Jamadar et al., 2010b; Figure 3).

There is evidence that preparatory switch-repeat sPPC activity occurs independently of the specific parameters of the two tasks being switched. Preparatory switch-repeat sPPC activation differences were found when shifting between either target spatial location or task rules (Slagter et al., 2006; Chiu and Yantis, 2009), and regardless of whether preparation involves only stimulus-set features or also incorporates response-set features (Ruge et al., in press). In the latter study, more posterior sections within PFC and PPC were related to stimulus-set preparation whereas more anterior sections of these areas were related to response-set preparation (Figure 4). However, the strength of PPC activation seems to be affected by the number of possible response choices and the number of task-sets. Forstmann et al. (2006) reported greater PPC activation for trials that afforded a choice among two or more tasks compared to trials that prescribed a specific task. Ruge et al. (2009a) found that preparatory activation in PPC was larger when a bivalent target was presented before the cue, possibly because the target activated both task-sets.

This selective review of fMRI findings leads to a set of tentative conclusions about the role of the fronto-parietal network in taskswitching. Preparatory and target-driven control processes associated with switching or repeating task-sets in cued-trials paradigms may be subserved by partly distinct PFC and PPC activity. In PFC, dorsal and anterior regions may subserve preparatory processes involved in goal-directed activation of task-specific areas, whereas more ventral and PM regions may subserve target-driven processes related to interference control and activation/implementation of unprepared task-sets. This pattern is consistent with models of functional organization in the PFC (Corbetta and Shulman, 2002; Koechlin and Summerfield, 2007). PPC activation under prepared task conditions may be related to advance preparation of attentional focus to relevant task rules, so that greater S-R activation in PPC is associated with reduced residual RT switch cost and larger cuelocked switch positivity.

Is there evidence for preparatory activity beyond fronto-parietal networks? Although most task-switching studies have focused on activation patterns in fronto-parietal networks, there is also increasing evidence for the role of the basal ganglia in preparatory processes. In concert with the cortex, the basal ganglia play a crucial role in controlling strategic motor behavior (Bogacz et al., 2010). However, few task-switching studies report activation within the basal ganglia. One possible key to explaining this puzzle is that neuroimaging studies have often focused on identifying regions that exhibit a greater transient increase of activity on switch trials following cue (or target) onset. This approach may overlook regions that exert sustained influences on flexibility (cf. Braver et al., 2003; Leber et al., 2009). Moreover, it is possible that brain areas can contribute to satisfying the need for flexibility while remaining insensitive to trial-by-trial variability in task demands. A recent fMRI study by Leber et al. (2009) provides evidence that activation in the subthalamic nucleus (STN) and putamen, among other cortical brain areas, predicted the behavioral switch costs of the upcoming trial without showing a significant difference between switch and repeat trials. This finding provides a compelling and parsimonious explanation for why the basal ganglia have not been prominent in previous neuroimaging studies. Moreover, the results show the predictive nature of brain activations for upcoming behavior (see also Bode and Haynes, 2009). Clearly, however, further work is necessary to understand how cortical and subcortical areas interact in providing flexible control of behavior.

In this section, we have focused largely on studies that do show differential BOLD activation for switch vs. repeat trials. However, it is important to note that many studies that report general trial-related or even preparation-related activation in prefrontal and parietal areas, do not find significant activation differences between switch and repeat trials (e.g., Luks et al., 2002; Brass and von Cramon, 2004; Forstmann et al., 2005; Ruge et al., 2005). A detailed analysis of methodological differences between studies that do and those that do not show switch-related activation is outside the scope of this focused review (see Figure 2B caption). In any case, the fact that many studies do show such an effect points to the relevance of additional modulatory variables that determine the strength of preparatory switch-repeat BOLD activation, and thus, might inspire more systematic research on this issue. One possible clue to explaining the lack of consistency of this finding may relate to individual differences in task-switching strategy (see Figure 3). Importantly, the striking discrepancy between highly consistent preparatory switch-repeat activation in ERP studies and the rather heterogeneous picture emerging from fMRI studies calls for more systematic future studies directly comparing ERP and fMRI results based on exactly the same experimental procedure (see Jamadar et al., 2010b; see Analyses of Behavior-Brain and Brain-Brain Linkages). Such multi-disciplinary studies can help capitalize on inter-individual and intra-individual variability across different measures to constrain the interpretation of processes reflected in ERP and fMRI indices of preparatory activation. We discuss some recent studies that use such an approach in the following section.

\section{FUTURE DIRECTIONS}

Our focused review highlights some key findings from ERP and fMRI methodologies about the timeline and localization of preparation processes. It also reveals significant gaps in knowledge about the nature of these processes. In the opening section, we argued that conventional behavioral measures (i.e., mean RTs and error rates) cannot provide direct measures of the multiple component processes contributing to advance preparation and task-set implementation. Recent behavioral studies have applied computational model analyses to derive model parameters that characterize underlying components of task-switching performance (e.g., Logan and Schneider, 2006; Barceló et al., 2008; Meiran et al., 2008). Although such studies have addressed some of the same questions as neuroscientific studies, the aim of this review is 
to examine what neuroscientific methodologies have added to our understanding of advance preparation processes and associated neural networks. Brain activation studies offer more direct measures of processes operating at different time points or in distinct brain areas. However, these measures are typically derived based on two implicit assumptions. One assumption is that automatic and strategic processes activated during advance preparation do not vary on a trial-by-trial basis. Hence, the same cognitive processes are assumed to be activated throughout the entire block of trials and to be represented in average ERP waveforms or average BOLD activations. The second assumption is that there is little or no variability in the relative reliance on automatic vs. strategic processes or the choice of specific strategic processes between individuals. Both assumptions are also implicit in the use of conventional behavioral measures that average over trials and individuals. We argue that both assumptions are likely wrong, as task-switching studies show considerable inter-trial and inter-individual variability in behavioral performance, as well as in ERP components and BOLD activation associated with advance preparation. Hence, in this section, we discuss two recent approaches that attempt to arrive at meaningful insights regarding how advance preparation is accomplished, by embracing rather than ignoring this variability.

\section{ANALYSES OF BEHAVIOR-BRAIN AND BRAIN-BRAIN LINKAGES}

What can the relationship between performance and neuroscientific measures tell us about the functional and structural organization of advance preparation? One approach capitalizes on variability across behavioral and brain imaging measures - it focuses on identifying underlying processes by examining relationships between behavioral, ERP and fMRI analyses. This approach has been helpful in explicating the functional significance of neuroscientific measures by linking variability in performance and brain activation measures. It has also been useful in addressing two questions that are relevant to theoretical formulations in the task-switching literature. First, is preparation to switch task an all-or-none process (De Jong, 2000) or can preparation vary in efficiency across trials? Second, is there any evidence for preparation processes that are elicited only on switch trials? Specifically, this approach seeks to establish brain-behavior (e.g., relationships between ERP components and behavior or fMRI activations and behavior) and brain-brain linkages (e.g., relationships between ERP components and fMRI activations) that quantitatively integrate these different measures.

In the ERP literature, these linkages have targeted both interindividual variability, by correlating the amplitude or latency of ERP components with behavioral measures (e.g., Karayanidis et al., 2009) and intra-individual variability, by averaging ERP trials equated for RT (e.g., Goffaux et al., 2006) or partitioning the ERP data into fast and slow RT trials (e.g., Lavric et al., 2008). The latter approaches capitalize on evidence that, in long CTI conditions, RT switch cost varies across the RT distribution (De Jong, 2000), so that fast trials show little or no switch cost (i.e., efficient preparation), whereas slow trials show a large switch cost, (i.e., absent or less efficient preparation). If this is related to variability in the strength of activation of strategic preparation processes on a trial-by-trial basis, ERP components associated with advance preparation should be elicited on fast RT (prepared) but not slow RT (unprepared) trials. In support of this prediction, Lavric et al. (2008) reported that a larger late frontal negativity for switch trials was associated with better preparation. Furthermore, the parietal switch positivity was present on fast trials only (i.e., when preparation was more effective). Goffaux et al. (2006) showed that differences in posterior cue-locked ERPs remain on trials equated for RT, indicating that these ERPs likely reflect differences in preparation for the upcoming trial, rather than generalized speed differences.

The presence of a parietal switch positivity for prepared but not unprepared trials (Lavric et al., 2008) is consistent with an all-ornone preparation processes. However, this partitioning method does not allow fine-grained examination of variation in cue-locked ERPs across the entire RT distribution. Karayanidis et al. (in press) used orthogonal polynomial regression analysis (Woestenburg et al., 1983) to extract single-trial cue-locked ERPs for each RT percentile. The amplitude of the posterior cue-locked positivity varied with RT percentile for switch but not repeat trials, showing that a larger positivity was associated with faster switch RTs. Notably, even the slowest switch trials had a larger cue-locked positivity than the fastest repeat trials, suggesting that all switch trials were associated with more strategic preparation than repeat trials (see also Goffaux et al., 2006). The amplitude of the later fronto-central pre-target negativity also varied with RT, being larger for fast than slow RT trials ${ }^{3}$. However, although the switch vs. repeat difference in cue-locked posterior positivity remained when controlling for RT, the difference in pre-target negativity was eliminated for trials with the same RT. These findings support a functional dissociation between the posterior positivity and fronto-central pre-target negativity. The former varies as a function of trial-by-trial variation in advance preparation on switch trials, consistent with stimulus-set shifting (Mueller et al., 2007, 2009; Astle et al., 2008). The latter varies as a function of more general preparation on both switch and repeat trials, consistent with anticipatory attention and response preparedness (e.g., Brunia, 1999).

Links between behavioral measures and strength of fMRI activity were reviewed in Section "Linking Fronto-Parietal Networks to Preparatory and Target-Driven Control Processes." Examination of brain-brain relationships by integrating ERP and fMRI data can provide information about cognitive processing that cannot be obtained using either method alone (Dale and Halgren, 2001). One approach has been to use areas of fMRI activation as potential generator sources in ERP dipole modeling (Swainson et al., 2003; Brass et al., 2005). An alternative approach is to examine variation in the strength of fMRI activation as a function of variation in ERP components that are associated with temporally and functionally distinct processes (e.g., Horovitz et al., 2002; Jamadar et al., 2010 b). This approach capitalizes on the temporal information provided by ERPs to constrain the interpretation of fMRI activations. Jamadar et al. (2010b; see Figure 3) used this approach to examine whether fMRI switch-repeat activation differences are associated with inter-individual variability in cue-locked or target-locked ERP components. Significant correlations were found between early and late preparatory components and localized switch-repeat activations. For example, there was a significant correlation between the switch-repeat contrast strength in SPPC and late cue-locked switch

${ }^{3}$ Note that this pre-target negativity is more clearly evident when using linked ears or linked mastoids reference. Its relationship to the slow frontal negativity seen with common average reference remains to be determined. 
positivity, in the absence of significant correlations between this contrast and other ERP components. While these ERP-fMRI correlations do not necessarily indicate that the location of the fMRI activity is the source of the switch positivity, they strongly suggest that activation in SPPC is associated with cue-locked preparation processes represented in the late switch positivity (Figure 3).

In summary, studies examining linkages among brain and behavior measures provide strong evidence in favor of a multi-component structure of advance preparation. The considerable variability in behavioral and neuroscientific measures of advance preparation, both across different participants and across trials for the same participant, strongly supports a graded rather than an all-or-none process. The finding that the relationship between behavioral and ERP variability held only for switch trials on some ERP components, but occurred for both trial types on other components, provides some support for the existence of both switch-specific and general preparation processes, at least in typical cued-trials paradigms.

\section{MODEL-BASED ANALYSES OF BEHAVIOR-BRAIN RELATIONSHIPS}

How do neuroscientific measures map onto decision-related psychological variables? The "model-based" approach accounts for the time course of, and individual differences in, preparatory processes by fitting quantitative mathematical models of latent psychological processes. This approach offers a more fine-grained analysis of behavior by providing model parameters that map onto latent processes contributing to the behavioral outcome. The association between model parameters that characterize the decision-process and neuroscientific measures provides evidence as to the functional and psychological relevance of the latter. This approach has been used in $\mathrm{fMRI}$ regression analysis to improve localization (e.g., Forstmann et al., 2008a,b, 2010) and in ERP regression analyses to provide converging evidence about the function of ERP components.

Intensive study of rapid choice in the single-task context using behavioral measures has led to a consensus that, after an initial stimulus encoding stage, a decision is made by accumulating evidence for each choice (see, e.g., Brown and Heathcote, 2008; Ratcliff and McKoon, 2008). Such models divide RT into two components: the time taken to select a response and the time taken for non-decision processes, such as stimulus encoding and response execution. Response selection depends on latent variables quantifying the rate of evidence accumulation and the threshold amount of evidence required to make a decision. Response choice corresponds to the first evidence total to exceed a threshold and choice RT to the time taken to accumulate that total. Equations for such models can be used to transform individual trial accuracy and RT data into estimates of model parameters, which in turn can be mapped onto both automatic (i.e., rate of evidence accumulation) and strategic (i.e., decision threshold) latent psychological processes for each participant. As these model parameters characterize fluctuations in the decision process and quantify individual differences (e.g., relative preference for accurate vs. speeded responding), they provide a quantitative characterization of both intra- and interindividual variability in performance.

Recently, these models have also been applied in the neuroscience domain to characterize the neural basis of decision making (see Gold and Shadlen, 2007; Bogacz et al., 2010). Forstmann et al. (2008b, 2010) applied this type of model-based approach in an fMRI context, showing that regressions using individual-participant model parameters enabled a clearer localization of areas involved in strategic (threshold setting) processes. In task-switching, Badre and Wagner (2006) introduced the control of associative memory during task-switching (CAM-TS) model, which was evaluated based on behavioral data. As has been done with evidence accumulation models, quantitative model parameters reflecting proactive interference were then directly related to switch-related brain activation. This approach showed that increased preparation was associated with reduced switch-repeat activation in mid-VLPFC but increased activation in iPPC (see also "Linking Fronto-Parietal Networks to Preparatory and Target-Driven Control Processes").

In the task-switching literature, performance costs associated with advance preparation and target-driven decision processes have been mapped onto different parameters of evidence accumulation models (Logan and Schneider, 2006; Karayanidis et al., 2009). This approach addresses one of the key weaknesses of behavioral data, by enabling a model-based decomposition of the time line and types of processes subserving overt responding. For example, non-decision time can provide a measure of how much cue-dependent preparation has occurred in the cue-to-target interval, with increased non-decision time indicating a greater delay in the initiation of the decision processes once the target appears, because preparation remains to be completed.

Consistent with this idea, Karayanidis et al. (2009, see Figure 5) found that, for fits of the EZ evidence accumulation model (Wagenmakers et al., 2007; Grasman et al., 2009), non-decision time varied as a function of the amount of information afforded by the cue. Specifically, non-decision time was smallest for both switch-to and repeat cues that provided certainty about the identity of upcoming task, increased for switch-away cues that provided certainty about a change in task but not the identity of the relevant task, and, to a greater degree, for non-informative cues that were followed by a task switch. Karayanidis et al. (2009) also found that cues predicting a certain task switch (i.e., switch-away and switch-to) resulted in a higher response threshold than cues predicting that task repetition was certain (i.e., repeat cues) or equiprobable (i.e., non-informative cues). The higher response threshold partially counteracted the tendency to make more errors on switch trials (by requiring the decision to be based on more evidence). Given that all four cue types were randomly presented, this suggests that participants engaged in cue-dependent trial-by-trial adjustment of response thresholds in order to balance demands for both fast and accurate responding. Higher thresholds also resulted in longer decision time; an effect that masked the shorter non-decision time for switch-away cues and resulted in a null difference in mean RT for switch-away trials relative to non-informative switch trials (i.e., non-informative cues that led to a switch trial). The latter had a shorter decision time due to a reduced response threshold. This null difference illustrates the insensitivity of the conventional mean RT measures to underlying process differences. In contrast, the model-based analysis integrates all aspects of choice behavior (e.g., error rates, RT variability and the speed of the fastest RTs, as well as mean RT), enabling greater sensitive to tradeoffs in underlying processes.

Karayanidis et al. (2009) looked at the relationship between individual variability in model parameters that theoretically can be set before target onset and variability in ERP components associated with advance preparation. In particular, the amplitude of the early 


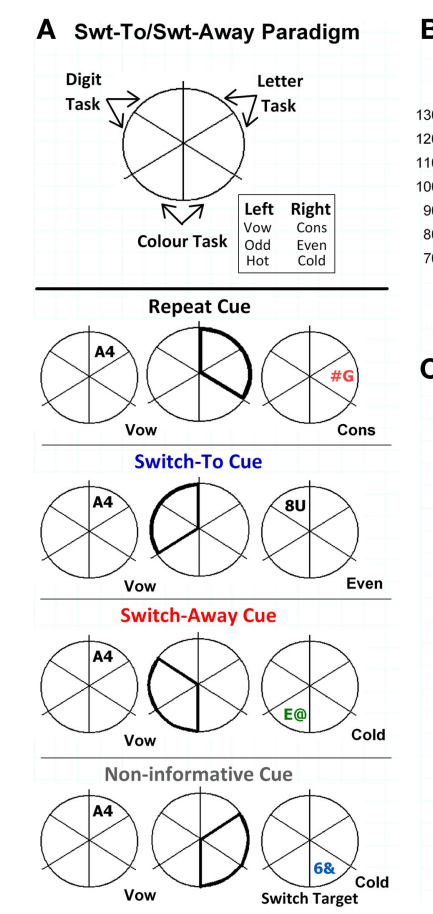

FIGURE 5 | From Karayanidis et al. (2009). (A) Participants $(n=24)$ used location based task cues to shift between three tasks. Cues could (a) validly predict a task repeat trial (repeat cues), (b) validly predict a shift away from the recently completed task-set and specify the upcoming task (switch-to), (c) validly predict a shift away from the recently completed task-set but leave task identity to target location (switch-away), or (d) be equally likely to be followed by a repeat or switch trial (e.g., non-informative cues leading to either a switch or repeat trial). (B) Switch-away cues resulted in more accurate but not faster responding than non-informative switch cues. Modeling of decision processes showed that this reflected differences in response criterion adjustment. Specifically, both cues that predicted a switch with certainty produced a higher response criterion than cues that predicted a certain (repeat) or likely (non-informative repeat or switch) repeat trial. This indicates trial-by-trial adjustment of response criterion depending on whether the cue provided certainty about an upcoming switch trial. These models also produce a non-decision time parameter that represents processes not directly related to the decision, such as stimulus processing and response execution. In task-switching paradigms, non-decision time is also affected by whether advance preparation has been effectively completed before target onset (see Karayanidis et al., 2009). In contrast to response criterion, non-decision time reduced progressively from non-informative switch to switch-away to switch-to trials, but did not differ between the latter and repeat trials. Hence, cues providing certainty of an upcoming shift away from the current task-set elicited some advance preparation, even when they did not define the new task-set. Interestingly, the decision time advantage of non-informative switch over switch-away trials (resulting from lower response criterion for the former) masked the non-decision time advantage of switchaway over non-informative switch trials (resulting from partial preparation), resulting in no net difference in mean RT. (C) Both switch-to and switch-away cues elicited an early cue-locked positivity that was not seen with either repeat or non-informative cues. (D) The amplitude of early cue-locked positivity was negatively correlated with RT, non-decision time and criterion for switch-to trials and with RT and non-decision time for switch-away trials. cue-locked positivity, which was elicited only by cues that validly predicted a task switch, was negatively correlated with non-decision time, suggesting that both measures index some aspect of advance preparation. That is, a larger early positivity was associated with greater advance preparation, resulting in shorter delay in the onset of decision processing. Additionally, early cue-locked positivity amplitude was related with lower response threshold for switch-to trials, supporting a relationship between degree of advance preparation and criterion setting (see also Karayanidis et al., 2010). While it is still too early for firm conclusions as to the strength and/or direction of the association between ERP components and decisionrelated parameters, this approach is clearly promising in terms of testing hypotheses about advance preparation processes ${ }^{4}$.

${ }^{4}$ Madden et al. (2009) used a similar approach correlating diffusion tensor imaging measures such as fractional anisotropy as an index of white matter integrity in young and old adults and evidence accumulation model parameters in a taskswitching paradigm.

\section{CONCLUSION}

In the present paper, we reviewed recent empirical evidence from the neurosciences on preparatory processes in task-switching. We showed that ERP and fMRI methodologies provide a window into when preparatory processes occur and where they take place in the human brain. In particular, different cue-locked ERP components, such as the cue-locked positivity and slow frontal negativity, provide direct measures of anticipatory control in relation to stimulus and response selection, respectively. fMRI studies have shown that areas within the fronto-parietal network implement both preparatory and target-driven control processes. In general, both methodologies are compatible with the conceptualization of advance preparation as a set of processes that are differentially activated for switch and/ or repeat trials dependent on individual variability, task parameters, and requirements. However, marked variability in these findings suggests that an important goal for future studies will be to account for inter-individual and intra-individual variations on advance preparation. To this end, we discussed recent studies that 
have attempted to use variability in behavioral performance and evidence accumulation models of decision processes to leverage the strengths of neuroscience methodologies. This approach may open the way for an integrated understanding of the "when," "where," and "how" of advance preparation in task-switching.

\section{REFERENCES}

Aron, A. R., Robbins, T. W., and Poldrack, R. A. (2004). Inhibition and the right inferior frontal cortex. Trends Cogn. Sci. (Regul. Ed.) 8, 170-177.

Astle, D. E., Jackson, G. M., and Swainson, R. (2006). Dissociating neural indices of dynamic cognitive control in advance task-set preparation: an ERP study of task switching. Brain Res. 1125, 94-103.

Astle, D. E., Jackson, G. M., and Swainson, R. (2008). Fractionating the cognitive control required to bring about a change in task: a dense-sensor event-related potential study. J. Cogn. Neurosci. 20, 255-267.

Badre, D., and Wagner, A. D. (2006). Computational and neurobiological mechanisms underlying cognitive flexibility. Proc. Natl. Acad. Sci. U.S.A. 18, 7186-7191.

Barceló, F., Periáñez, J. A., and Nyhus, E. (2008). An information theoretical approach to task-switching: evidence from cognitive brain potentials in humans. Front. Hum. Neurosci. 1:13. doi: 10.3389/neuro.09.013.2007.

Bode, S., and Haynes, J.-D. (2009). Decoding sequential stages of task preparation in the human brain. Neuroimage 45, 606-613.

Bogacz, R., Wagenmakers, E.-J., Forstmann, B. U., and Nieuwenhuis, S. (2010). The neural basis of the speedaccuracy tradeoff. Trends Neurosci. 33, $10-16$.

Brass, M., Ullsperger, M., Knoesche, T. R., von Cramon, D. Y., and Phillips, N. A. (2005). Who comes first? The role of the prefrontal and parietal cortex in cognitive control. J. Cogn. Neurosci. 17, 1367-1375.

Brass, M., and von Cramon, D. Y. (2004). Decomposing components of task preparation with functional magnetic resonance imaging. J. Cogn. Neurosci. 16, 609-620.

Braver, T. S., Reynolds, J. R., and Donaldson, D. I. (2003). Neural mechanisms of transient and sustained cognitive control during task switching. Neuron 39, 713-726.

Braver, T. S., and Ruge, H. (2006). "Functional neuroimaging of executive functions," in Handbook of Functional Neuroimaging of Cognition, eds R. Cabeza and A. Kingstone, Cambridge, MA: (MIT Press), 307-347.

Brown, S. D., and Heathcote, A. (2008). The simplest complete model of choice reaction time: linear ballistic accumulation. Cogn. Psychol. 57, 153-178.

Brunia, C.H.M. (1999). Neural aspects of anticipatory behaviour. Acta Psychol. (Amst) 101, 213-242.

Bunge, S. A., Kahn, I., Wallis, J. D., Miller, E. K., and Wagner,A. D. (2003). Neural circuits subserving the retrieval and maintenance of abstract rules. $J$. Neurophysiol. 90, 3419-3428.

Chiu, Y.-C., and Yantis, S. (2009). A domain-independent source of cognitive control for task sets: shifting spatial attention and switching categorisation rules. J. Neurosci. 29, 3930-3938.

Corbetta, M., and Shulman, G. L. (2002). Control of goal-directed and stimulusdriven attention in the brain. Nat. Rev. Neurosci. 3, 201-215.

Crone, E. A., Wendelken, C., Donohue, S. E., and Bunge, S. A. (2006). Neural evidence for dissociable components of task-switching. Cereb. Cortex 16, 475-486.

Dale, A. M., and Halgren, E. (2001). Spatiotemporal mapping of brain activity by integration of multiple imaging modalities. Curr. Opin. Neurobiol. 11, 202-208.

De Jong, R. (2000). "An intention - activation account of residual switch costs," in Attention and Performance XVIII, eds S. Monsell and J. Driver (Cambridge, MA: MIT Press), 357-376.

Dove, A., Pollmann, S., Schubert, T., Wiggins, C. J., and von Cramon, D. Y. (2000). Prefrontal cortex activation in task switching: an event-related fMRI study. Cogn. Brain Res. 9, 103-109.

Forstmann, B. U., Brass, M., Koch, I., and von Cramon, D. Y. (2005). Internally generated and directly cued task sets: an investigation with fMRI. Neuropsychologia 43, 943-952.

Forstmann, B. U., Brass, M., Koch, I., and von Cramon, D. Y. (2006). Voluntary selection of task sets reveal by functional magnetic resonance imaging. J. Cogn. Neurosci. 18, 388-398.

Forstmann, B. U., Brown, S., Dutilh, G., Neumann, J., and Wagenmakers, E.-J. (2010). The neural substrate of prior making: a model-based analysis. Front. Hum. Neurosci. 4:40. doi: 10.3389/ fnhum.2010.00040

Forstmann, B. U., Jahfari, S., Scholte, H. S., Wolfensteller, U., van den Wildenberg, W. P. M., and Ridderinkhof, K. R. (2008a). Function and structure of information in perceptual decision

\section{ACKNOWLEDGMENTS}

This work was supported by VENI (B. U. Forstmann) and a University of Newcastle Career Fellowship (F. Karayanidis). We thank Pat Michie for comments on an earlier draft and Lisa Whitson for assistance with manuscript preparation.

the right inferior frontal cortex predict individual differences in response inhibition: a model-based approach. $J$ Neurosci. 28, 9790-9796.

Forstmann, B. U., Dutilh, G., Brown, S., Neumann, J., von Cramon, D. Y. Ridderinkhof, K.R., andWagenmakers, E.-J. (2008b). Striatum and pre-SMA facilitate decision-making under time pressure. Proc. Natl. Acad. Sci. U.S.A. 105, 17538-17542.

Goffaux, P., Phillips, N. A., Sinai, M. and Pushkar, D. (2006). Behavioural and electrophysiological measures of task switching during single and mixed-task conditions. Biol. Psychol. 72, 278-290.

Gold, J. I., and Shadlen, M. N. (2007). The neural basis of decision making. Annu. Rev. Neurosci. 30, 535-574.

Grasman, R. P. P. P., Wagenmakers, E.-J. and van der Maas, H. L. J. (2009). On the mean and variance of response times under the diffusion model with an application to parameter estimation. J. Math. Psychol. 53, 55-68.

Hommel, B., and Colzato, L. S. (2010). Games with(out) Frontiers: toward an integrated science of human cognition. Front. Psychol. 1:2. doi: 10.3389/ fpsyg.2010.00002.

Horovitz, S. G., Skudlarski, P., and Gore, J C. (2002). Correlations and dissociations between BOLD signal and P300 amplitude in an auditory oddball task: a parametric approach to combining $\mathrm{fMRI}$ and ERP. Magn. Reson. Imaging 20, 319-325.

Hsieh, S., and Yu, Y.-T. (2003). Switching between simple response-sets: inferences from the lateralised readiness potential. Cogn. Brain Res. 17, 228-237.

Jamadar, S., Michie, P. T., and Karayanidis, F. (2010a). Sequence effects in cued task switching modulate response preparedness and repetition priming processes. Psychophysiology 47 365-386.

Jamadar, S., Hughes, M., Fulham, W. R., Michie, P. T., and Karayanidis, F. (2010b). The spatial and temporal dynamics of anticipatory preparation and response inhibition in taskswitching. Neuroimage 51, 432-449.

Jennings, J. R., and van der Molen, M. W. (2005). Preparation for speeded action as a psychophysiological concept. Psychol. Bull. 131, 434-459.

Jost, K., Mayr, U., and Rosler, F. (2008). Is task switching nothing but cue prim- ing? Evidence from ERPs. Cogn. Affect. Behav. Neurosci. 8, 74-84.

Karayanidis, F., Coltheart, M., Michie, P. T., and Murphy, K. (2003). Electrophysiological correlates of anticipatory and post-stimulus components of task-switching. Psychophysiology 40, 329-348.

Karayanidis, F., Mansfield, E.L., Galloway, K. L., Smith, J., Provost, A., and Heathcote, A. (2009). Anticipatory reconfiguration elicited by fully and partially informative cues that validly predict a switch in task. Cogn. Affect. Behav. Neurosci. 9, 202-215.

Karayanidis, F., Provost, A., Brown, S., Paton, B., and Heathcote, A. (in press). Switch-specific and general preparation map onto different ERP components in a task-switching paradigm. Psychophysiology.

Karayanidis, F., Whitson, L. R., Michie, P. T., and Heathcote, A. (2010). Neural correlates and temporal dynamics of task-switching in normal aging. In W. Christensen, E. Schier, and J. Sutton (eds.), ASCS09: Proceedings of the 9 th Conference of the Australasian Society for Cognitive Science (pp. 170-177). Sydney: Macquarie Centre for Cognitive Science. doi: 10.5096/ASCS200926 URL: http:// www.maccs.mq.edu.au/news/ conferences/2009/ASCS2009/html/ karayanidis.html

Kieffaber, P. D., and Hetrick, W. P. (2005). Event-related potential correlates of task switching and switch costs. Psychophysiology 42, 56-71.

Kiesel, A., Steinhauser, M., Wendt, M., Falkenstein, M., Jost, K., Philipp, A., and Koch, I. (in press). Control and interference in task-switching - a review. Psychol. Bull.

Kimberg, D. Y., Aguirre, G. K., and D’Esposito, M. (2000). Modulation of task-related neural activity in taskswitching: an fMRI study. Cogn. Brain Res. 10, 189-196.

Koechlin, E., and Summerfield, C. (2007). An information theoretical approach to prefrontal executive function. Trends Cogn. Sci. (Regul. Ed.) 11, 229-235.

Lavric, A., Mizon, G., and Monsell, S. (2008). Neurophysiological signature of effective anticipatory task-set control: a task-switching investigation. Eur. J. Neurosci. 28, 1016-1029.

Leber, A. B., Turk-Browne, N. B., and Chun, M.M.(2009). Neural predictors of moment-to-moment fluctuations 
in cognitive flexibility. Proc. Natl. Acad. Sci. U.S.A. 105, 13592-13597.

Logan, G. D., and Bundesen, C. (2003). Clever homunculus: is there an endogenous act of control in the explicit task-cuing procedure? J. Exp. Psychol. Hum. Percept. Perform. 29, 575-599.

Logan, G. D., and Schneider, D. W. (2006). Priming or executive control? Associative priming of cue encoding increases "switch costs" in the explicit task-cuing paradigm. Mem. Cognit. 34, 1250-1259.

Logothetis, N. K. (2002). The neural basis of the blood-oxygen-level-dependent functional magnetic resonance imaging signal. Philos. Trans. R. Soc. Lond., B, Biol. Sci. 357, 1003-1037.

Luks, T. L., Simpson, G. V., Feiwell, R. J., and Miller, W. L. (2002). Evidence for anterior cingulate cortex involvement in monitoring preparatory attentional set. Neuroimage 17, 792-802.

Madden, D. J., Spaniol, J., Costello, M. C., Bucur, B., White, L. E., Cabeza, R., Davis, S. W., Dennis, N.A., Provenzale, J.M., and Huettel, S.A. (2009). Cerebral white matter integrity mediates adult age differences in cognitive performance. J. Cogn. Neurosci. 21, 289-302.

Meiran, N., Chorev, Z., and Sapir, A. (2000). Component Processes in Task Switching. Cogn. Psychol. 41, 211-253.

Meiran, N., Kessler, Y., and Adi-Japha, E. (2008). Control by action representation and input selection (CARIS): a theoretical framework for task switching. Psychol. Res. 72, 473-500.

Miniussi, C., Marzi, C. A., and Nobre, A. C. (2005). Modulation of brain activity by selective task sets observed using event-related potentials. Neuropsychologia 43, 1514-1528.

Mueller, S. C., Swainson, R., and Jackson, G. M. (2007). Behavioural and neurophysiological correlates of bivalent and univalent responses during task switching. Brain Res. 1157, 56-65.

Mueller, S. C., Swainson, R., and Jackson, G. M. (2009).ERP indices of persisting and current inhibitory control: a study of saccadic task switching. Neuroimage 45, 191-197.

Nicholson, R., Karayanidis, F., Davies, A., and Michie, P.T. (2006a).Components of task-set reconfiguration: differential effects of 'switch-to' and 'switch-away' cues. Brain Res. 1121, 160-176.

Nicholson, R., Karayanidis, F., Bumak, E., Poboka, D., and Michie, P. T. (2006b). ERPs dissociate the effects of switching task sets and task cues. Brain Res. 1095, 107-123.

Perianez, J. A., and Barcelo, F. (2009). Updating sensory versus task representations during task-switching: insights from cognitive brain potentials in humans. Neuropsychologia 47, 1160-1172.

Pessoa, L., Rossi, A., Japee, S., Desimone, R., and Ungerleider, L. G. (2009). Attentional control during the transient updating of cue information. Brain Res. 1247, 149-158.

Poulsen, C., Luu, P., Davey, C., and Tucker D. M. (2005). Dynamics of task sets: evidence from dense-array eventrelated potentials. Cogn. Brain Res. 24, 133-154.

Ratcliff, R., and McKoon, G. (2008). The diffusion decision model: theory and data for two-choice decision tasks. Neural. Comput. 20, 873-922.

Rogers, R. D., and Monsell, S. (1995). Costs of a predictable switch between simple cognitive tasks. J. Exp. Psychol. 124, 207-231.

Ruge, H., Brass, M., Koch, I., Rubin, O. Meiran, N., and von Cramon, D. Y. (2005). Advance preparation and stimulus-induced interference in cued task switching: further insights from BOLD fMRI. Neuropsychologia $43,340-355$.

Ruge, H., Braver, T., and Meiran, N. (2009a). Attention, intention and strategy in preparatory control. Neuropsychologia 47, 1670-1685.

Ruge, H., Goschke, T., and Braver, T. S. (2009b). Separating event-related BOLD components within trials: the partial-trial design revisited. Neuroimage 47, 501-513.

Ruge, H., Mueller, S. C., and Braver, T. S. (in press). Anticipating the consequences of action: an fMRI study of intention-based task preparation. Psychophysiology. DOI: 10.1111/ j.1469-8986.2010.01027.x. Available at http://0-www3.interscience.wiley. com.library.newcastle.edu.au/cgi-bin/ fulltext/123421972/PDFSTART
Ruge, H., Stoet, G. T., and Naumann, E. (2006). Attentional set mixing: effects on target selection and selective response activation. Psychophysiology 43, 413-421.

Rugg, M. D., and Coles, M. G. H. (1995). Electrophysiology of Mind: Event-Related Brain Potentials and Cognition. New York: Oxford University Press.

Rushworth, M. F., Hadland, K. A., Gaffan, D., and Passingham, R. E. (2003). The effect of cingulate cortex lesions on task switching and working memory. J. Cogn. Neurosci. 15, 338-353.

Rushworth, M. F. S., Hadland, K. A. Paus, T., and Sipila, P. K. (2002). Role of the human medial frontal cortex in task switching: a combined $\mathrm{AMR}$ and TMS study. J. Neurophysiol. 87, 2577-2592.

Sakai, K., and Passingham, R. E. (2006) Prefrontal set activity predicts rulespecific neural processing during subsequent cognitive performance. $J$. Neurosci. 26, 1211-1218.

Slagter,H.A., Weissman, D. H., Giesbrecht, B., Kenemans, J. L., Mangun, G. R. Kok, A., and Woldorff, M. G. (2006) Brain regions activated by endogenous preparatory set shifting as revealed by fMRI. Cogn. Affect. Behav. Neurosci. 6 175-189.

Sohn, M., Ursu, S., Anderson, J. R., Stenger V. A., and Carter, C. S. (2000). The role of prefrontal cortex and posterior parietal cortex in task-switching. Proc. Natl. Acad. Sci. U.S.A. 97, 13448-13453.

Swainson, R., Cunnington, R., Jackson, G. M., Rorden, C., Peters, A. M., Morris, P. G., and Jackson, S. R. (2003). Cognitive control mechanisms revealed by ERP and fMRI: evidence from repeated task-switching. J. Cogn. Neurosci. 15, 785-799.

Swainson, R., Jackson, S. R., and Jackson, G.M. (2006). Using advance information in dynamic cognitive control: an ERP study of task-switching. Brain Res. 1105, 61-72.

Wagenmakers, E.-J., van der Maas, H. L. J., and Grasman, R. P. P. P. (2007) An EZ-diffusion model for response time and accuracy. Psychon. Bull. Rev. 14, 3-22.
Wager, T. D., Jonides, J., and Reading, S. (2004). Neuroimaging studies of shifting attention: a meta-analysis. Neuroimage 22, 1679-1693.

Woestenburg, J. C., Verbaten, M. N., Van Hees, H. H., and Slangen, J. L. (1983). Single trial ERP estimation in the frequency domain using orthogonal polynomial trend analysis (OPTA): estimation of individual habituation. Biol. Psychol. 17, 173-191.

Wylie, G., and Allport, A. (2000). Task switching and the measurement of 'switch costs'. Psychol. Res. 63, 212-233.

Wylie, G. R., Javitt, D. C., and Foxe, J. J. (2006). Jumping the gun: is effective preparation contingent upon anticipatory activation in task-relevant neural circuitry? Cereb. Cortex 16, 394-404.

Wylie, G. R., Murray, M. M., Javitt, D. C., and Foxe, J. J. (2009). Distinct neurophysiological mechanisms mediate mixing costs and switch costs. J. Cogn. Neurosci. 21, 105-118.

Conflict of Interest Statement: The authors declare that the research was conducted in the absence of any commercial or financial relationships that could be construed as a potential conflict of interest.

Received: 16 March 2010; paper pending published: 31 March 2010; accepted: 07 June 2010; published online: 15 July 2010. Citation: Karayanidis F, Jamadar S, Ruge $H$, Phillips N, Heathcote A and Forstmann $B U$ (2010) Advance preparation in taskswitching: converging evidence from behavioral, brain activation, and model-based approaches. Front. Psychology 1:25. doi: 10.3389/fpsyg.2010.00025

This article was submitted to Frontier in Cognition, a specialty of Frontiers in Psychology.

Copyright (C) 2010 Karayanidis, Jamadar, Ruge, Phillips, Heathcote and Forstmann. This is an open-access article subject to an exclusive license agreement between the authors and the Frontiers Research Foundation, which permits unrestricted use, distribution, and reproduction in any medium, provided the original authors and source are credited. 